\title{
Membrane nanotubes: Novel communication between distant cells
}

\author{
ZHANG JiangHui \& ZHANG YouYi* \\ Institute of Vascular Medicine of Peking University Third Hospital, Key Laboratory of Cardiovascular Molecular Biology and Regulatory \\ Peptides of Ministry of Health, Key Laboratory of Molecular Cardiovascular Sciences of Ministry of Education and Beijing Key Laboratory of \\ Cardiovascular Receptors Research, Beijing 100191, China
}

Received June 6, 2013; accepted July 24, 2013; published online September 3, 2013

\begin{abstract}
The many kinds of cell structures involved in cell-cell communication include tight junction, adherens junction and gap junction, but almost all are between adjacent cells. Recently, a general and dynamic membrane tether, termed tunneling nanotubes or membrane nanotubes (MNTs), was discovered to be involved in communication between distant cells. By facilitating intercellular communication, MNTs contribute to many biological functions and pathologic changes in cells. Many works have revealed the structure, formation and functional properties of MNTs. However, as novel structures, further research is needed.
\end{abstract}

membrane nanotubes, microstructure, physiological functions, cell communication

Citation: Zhang J H, Zhang Y Y. Membrane nanotubes: Novel communication between distant cells. Sci China Life Sci, 2013, 56: 994-999, doi: $10.1007 / \mathrm{s} 11427-013-4548-3$

Membrane nanotubes (MNTs) are line-like membrane tubes that connect two distant cells (Figure 1). The structure has been observed in many eukaryotic cells, such as rat tumor, natural killer (NK), and endothelial progenitor cells [1-4]. Recently, we described the structure between cardiomyocytes (CMs) and cardiofibroblasts (FBs) and found MNT-like bridges in myocardial tissue of mice (Figure 2) [5]. Other groups found MNTs between putative dendritic cells in mouse cornea and in solid tumors in humans [6,7]. Hence, MNTs may be widespread among animal cells both in vitro and in vivo. MNTs are considered a neotype of cell communication, transporting many components and signals [8]. Clarifying the structure, function and detailed mechanisms of the formation of MNTs will help in better understanding the development and homeostasis of multicellular organisms. In this review, we elaborate current research into MNTs.

*Corresponding author (email: zhangyy@bjmu.edu.cn)

\section{Structural characteristics of MNTs}

In 2000, the graduate student Amin Rustom, using a fluorescence microscope, noticed some straight lines connecting rat tumor cells but mistook them for scratches on the plate. Four years later, by 3D live-cell microscopy, he identified the lines between rat pheochromocytoma PC12 cells as intercellular structures and called them MNTs (Figure 1A) [1]. At the same time, similar structures were found to connect immune cells [9]. The following study clarified the structure of MNTs.

\subsection{Morphology of MNTs}

Scanning electron microscopy revealed that the surface of MNTs, as ultrafine membranous channels, showed a seamless transition to the surface of the two connected cells (Figure 1B) [1]. The diameter of MNTs ranges from 50 to $200 \mathrm{~nm}$ and some are as long as the diameter of some cells [1]. In human monocyte-derived macrophages, most of the thicker MNTs $(>0.7 \mu \mathrm{m})$ contain microtubules, and in NK 

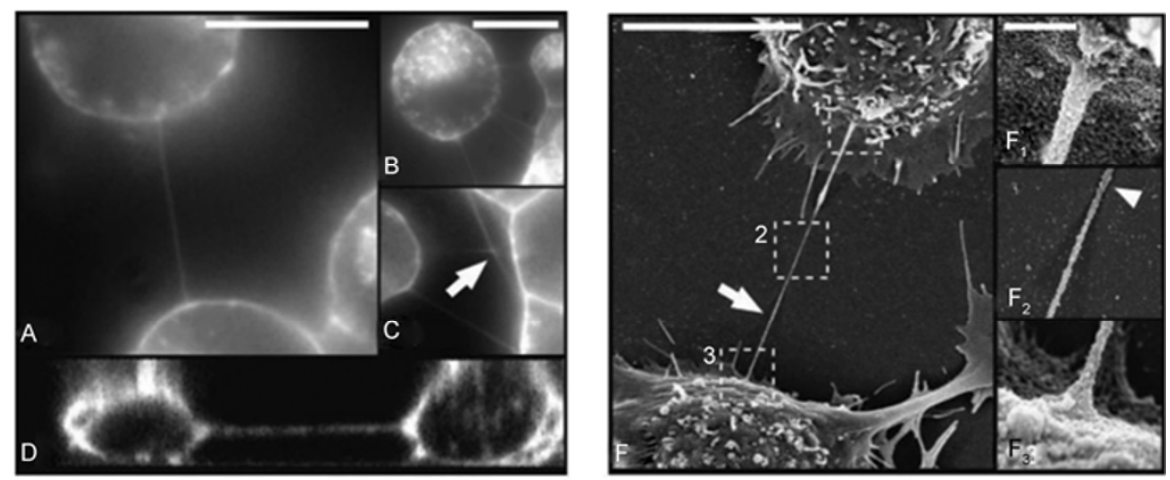

Figure 1 Structure of membrane nanotubes (MNTs) between PC12 cells. A-D, 3-D live-cell microscopy of the architecture of MNTs. Cells are connected with surrounding cells via one (A) or several MNTs ((B) and (C)); branched MNTs are observed (arrow). F, Scanning electron microscopy of ultrastructure of MNTs. The ultrastructure of PC12 cells with one MNT (F); for box areas, high magnification images are shown $\left(\mathrm{F}_{1}-\mathrm{F}_{3}\right)$ [1] (with permission from Science).

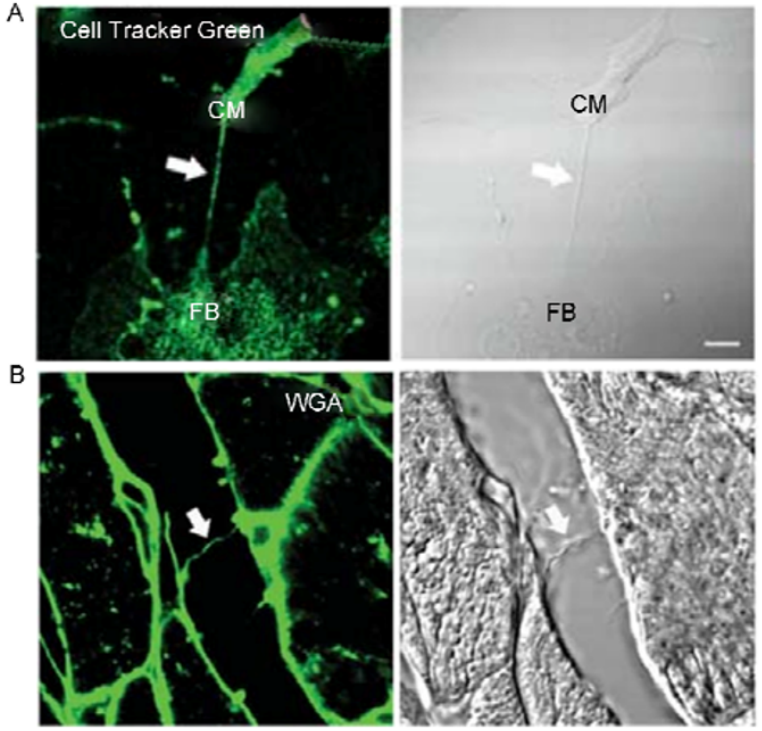

Figure 2 MNTs between rat ventricular cardiomyocytes (CMs) and cardiofibroblasts (FBs), and the MNTs-like structures in adult mouse heart tissue. A, MNTs (arrows) between CMs and FBs. B, Structures similar to MNTs in adult mouse heart tissue. The cells and tissue were stained by Alexa Fluor 488-conjugated wheat germ agglutinin (WGA) [5].

cells, the mean length of tubules per cells is inversely related to the MNT number [10,11]. These findings suggest that their thickness and length might be related to their components and number.

MNTs do not connect with any other substrate, and the ends of MNTs connect with the surface of cells. Although the microstructure of the ends has not been clarified, an open- or closed-ended MNT is formed depending on whether the end of the MNT fuses with the connecting cell membrane. As for open-ended MNTs, a transient pore is formed at the tip of MNTs and some intercellular cargo or larger organelles such as mitochondria or vesicles may be transported from the MNTs to the target cell via the pore $[1,4,12]$. Although there is no "open gate" at the tip of the close-end MNTs, membranous cargo can enter into the target cell by endocytic forces [4].

\subsection{Components of MNTs}

As for the construction of cell membranes, MNTs contain microtubules and F-actin, important cytoskeleton components $[5,13]$. F-actins but not microtubules are found in all MNTs connecting cells such as PC12 cells or myeloid cells [4]. Although F-actin is considered as the hallmark of MNTs, some reports indicated that not all MNTs between immature hippocampal neurons and astrocytes contain F-actin, and more interestingly, F-actin-lacking MNTs were longer than those containing F-actin [14].

Besides these cytoskeleton components, connexin 43, the most widely expressed gap-junction protein, is also found in the MNTs between some cells, such as normal rat kidney and neural crest cells [15]. Connexin 43 could mediate the formation of a gap-junction vesicle and allow the components to enter connected cells by endocytosis [12]. However, we did not observe connexin 43 in the MNTs between CMs and FBs, which indicated that other kinds of junction proteins might integrate into the MNT structure. Additionally, major histocompatibility complex class I chain-related protein A (MICA), DNAX-activating protein of $10 \mathrm{kD}$ and Vav-1 were observed at the submicron-scale junction of MNTs connecting NK cells and target cells. These proteins are related to activation of NK cells and lysis of target cells [13].

\section{Formation of MNTs}

Researchers have used cell culture models to uncover how MNTs are formed and the mechanisms are involved, which will help us better understand the biological meaning of MNTs. 


\subsection{Formation modes of MNTs}

Time-lapse imaging has shown that MNTs can be formed within a few minutes by two different modes: dislodgement of connected cells and outgrowth of a filopodium-like protrusion [4], as show in Figure 3. The methods may vary by cell type. Indeed, mobile cells, such as normal rat kidney and $\mathrm{T}$ cells, which easily connect with other cells, form tubes by the first method $[15,16]$. Most immobile cell types, such as PC12, form tubes by use of an outgrowth [17].

\subsection{Mechanisms involved in the formation of MNTs}

We divided the mechanisms of MNT formation into two types: MNT-promoting and -inhibiting mechanisms (Figure 4).

Many promoting mechanisms were studied in the immune system. The accumulation of MICA was observed at the end of the formation of MNTs between NK cells and mouse P815 cells, and overexpression of MICA in P815 cells induced the formation of MNTs [13]. Recently, overexpression of CD2 but not actin-interaction protein myosin IIA would induce the formation of MNTs between CD2 ${ }^{-}$ NK cells YTS and their target, 712 cells, and then increased the cytotoxic activity of YTS [11]. Additionally, the Fas signaling pathway could induce MNT formation by activating Rho GTPase in T cells [18]. Besides these in vitro studies, in adult mouse cornea, inflammation and serum starvation could promote MNT formation between histocompatibility complex class II+ cells [19]. Some studies investigated neural and cancerous systems. Between rat hippocampal neurons and astrocytes, activation of the P53/AKT/PI3K/ mammalian target of rapamycin channel by $\mathrm{H}_{2} \mathrm{O}_{2}$ increased the development of MNTs [20]. Recently, p53/caspase 3 activation led to cleavage of small calcium-binding protein S100A4 and subsequent decreased concentration; the resulting gradient of cells with low to high concentration of S100A4 provided direction for MNT formation [21]. M-sec/ Ral/exocyst, the filopodium-dependent mechanism, could promote the formation of MNTs in cervical cancer HeLa

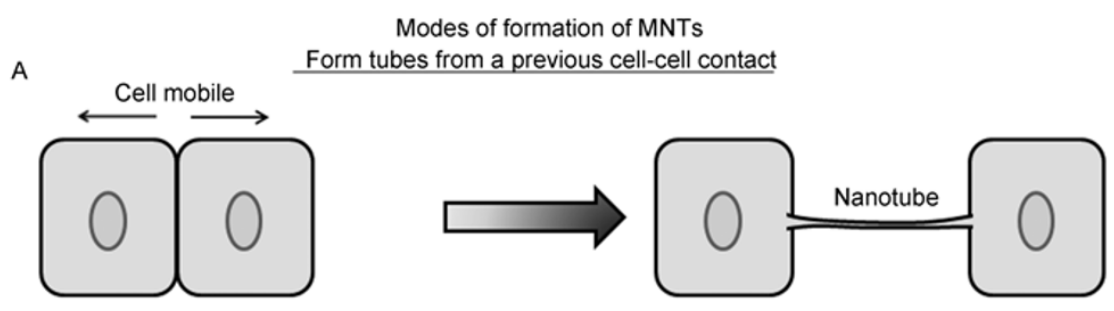

B Form tubes by extending tubes toward distant cells

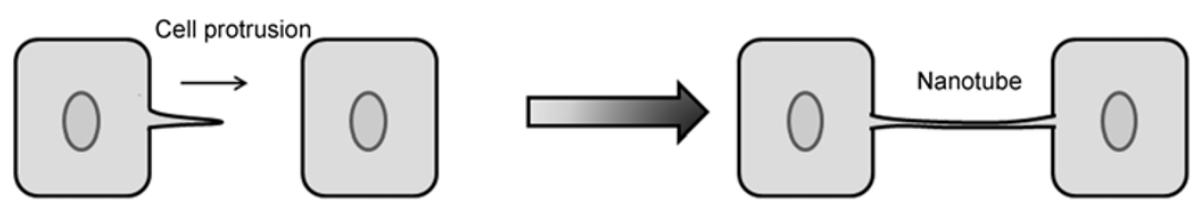

Figure 3 Two modes of formation of MNTs. A, Previous cell-cell contact. B, Formation of filopodium-like protrusion.

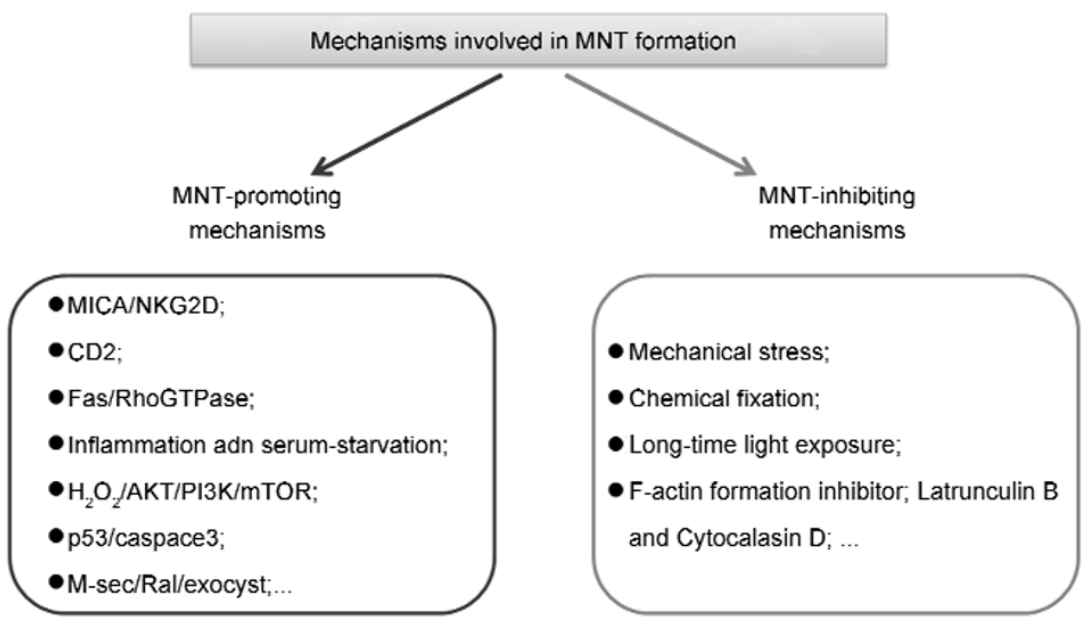

Figure 4 The mechanisms involved in regulating MNT formation. 
cells [22].

Our understanding of the MNT-inhibiting mechanism in the formation of MNTs is limited. As a thin tube-like structure, the presence of MNTs is not very stable. The organelles can be disrupted by mechanical stress, chemical fixation, and even long-term light exposure [12]. Latrunculin B and Cytocalasin D could inhibit the formation of MNTs $[17,23]$ and prevent the polymerization of actin filaments by binding to actin monomers or capping the F-actin plus end. Therefore, F-actin might have an important role in the formation of MNTs and may regulate MNT formation by affecting cytoskeleton remodeling.

\section{Biological significance of MNTs}

Many studies have investigated the biological function of this novel structure connecting two distant cells. Use of fluorescent proteins and/or dyes confirmed that MNTs could mediate the transportation of signals and components between distant cells and affect the cells' biological functions.

\subsection{Transport of organelles and signals}

MNTs were found to transport $\mathrm{Ca}^{2+}$, an important signal factor, between immune cells [2]. Recently, electrical coupling was found transferred between immature hippocampal neurons and astrocytes, and the transfer ability decreased with neuron maturation [14]. The gap-junction channel in MNTs was traditionally considered a gate for transfer of electrical coupling [12,15]. However, we found that $\mathrm{Ca}^{2+}$ could be transported through connexin 43-negative MNTs between $\mathrm{CMs}$ and FBs [5], so $\mathrm{Ca}^{2+}$ might be transported other than by a gap junction.

Besides electrical signals, bacteria, HIV and quantum dot can be transported by MNTs between mesenchymal stem/stromal cells (MSCs) or immune cells [10,16,24,25], and in our study, mitochondria and intercellular vesicles could be transported via MNTs between CMs and FBs (Figure 5) [5]. ATP and motor protein kinesin and dynein are required for the cargo transfer [10,26,27]. Recently, MNTs were found to transport porous silicon microparticles, so they may be involved in dispersion of therapeutic agents at the sites of diseased tissue [28].

The molecules in MNTs are transferred bi- or unidirectionally, probably depending on the cell type. Cellular components are transferred efficiently from rat renal tubular cells to bone-marrow multipotent MSCs [29]. Mitochondria could be transported via MNTs between progenitor and endothelial cells and between cardiac myocytes and cardiac fibroblasts [5,30]. Additionally, bi-directional transfer could shift to unidirectional transfer with specific stimulation, such as stress signals from damaged organelles [30].

\subsection{Spread of pathogens}

Within an infected host, viruses can disseminate by two mechanisms: (i) cell-free spread and (ii) direct cell-cell transfer, the latter being more efficient. The Mycobacterium bovis bacillus Calmette-Guérin could be transferred along the thin MNT surface toward macrophage cell bodies [10]. Besides bacteria, HIV-1 could be transferred from infected to uninfected $\mathrm{T}$ cells via MNTs [16]. This intercellular transmission of bacteria and virus is functionally important for the spread of pathogens, and the rearrangement of host-cell cytoskeletal proteins may be involved [31,32].

\subsection{Promotion of cell biological functions}

The cognate receptor/ligand pairs-MICA/NKG2D-
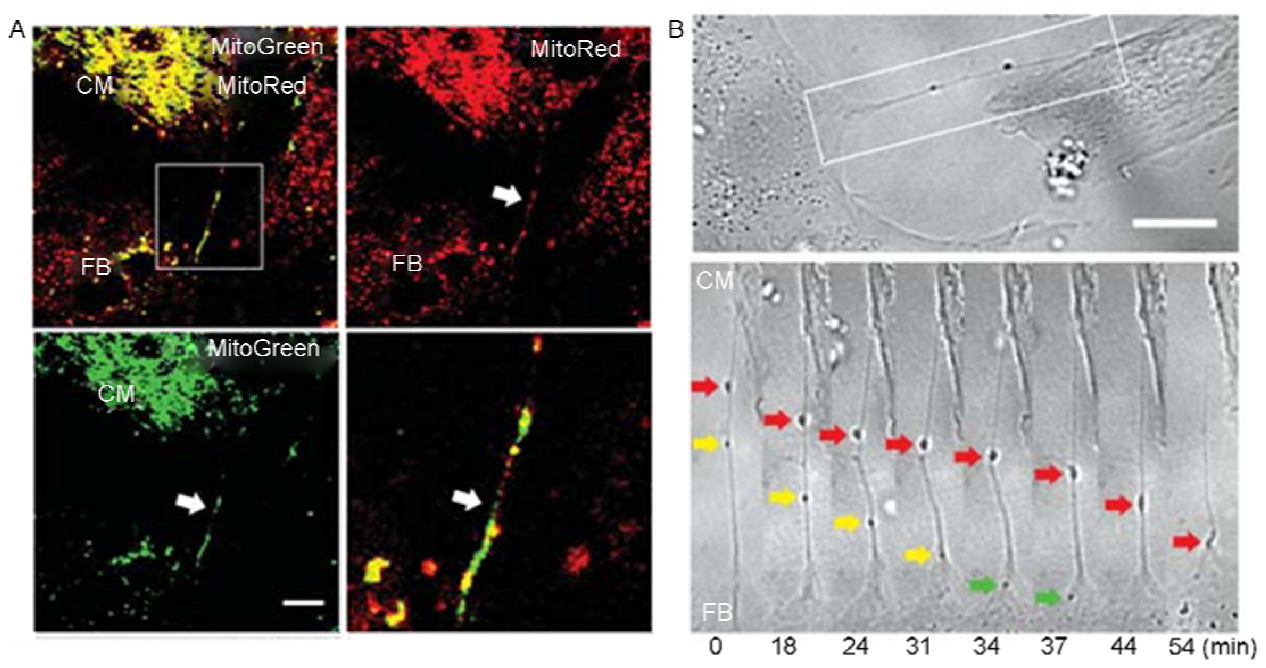

Figure 5 Cargo transported by MNTs (box) between CMs and FBs. A, Transfer of mitoTracker-labelled mitochondria. B, A snapshot of a time-lapse movie of two vehicles moving from a beating CM to a FB through an MNT (box). Red arrows point to vehicle 1 . Yellow arrows point to vehicle 2 moving within the MNTs and finally incorporated into the FB (green arrows) [5]. 
formed in MNTs between NK cells and target cells, can active NK cells and promote NK cell-mediated cytotoxicity [13]. Recently, MNTs observed between MSCs and vascular smooth muscle cells promoted the proliferation but not differentiation of MSCs by transfer of mitochondria [23], and MNTs between osteoclast precursors were found essential for osteoclastogenesis [33]. MNTs also exist between malignant tumor cells of mesothelioma and lung cancer and might play a critical role in tumor formation and development [7].

\section{Perspectives}

Although we have made great progress in the study of MNTs, many questions remain unanswered. The occurrence of MNTs has been observed in vitro and vivo. However, the specific MNTs markers have not been found. Protein screening might help us to further uncover the components and identify the markers of MNTs. Finding the specific markers will promote the elucidation of molecular mechanisms regulating the formation of MNTs as well as visualization and identification of these structures in vitro and in vivo.

With the development of fluorescent or electron microscopes, we can get more information about the ultrastructure and components of MNTs in deferent type of cells and then study whether these factors are related to MNTs' various functions.

As discussed here, MNTs play an important role in many pathophysiological processes by transporting some molecular signals. The technique of live cell imaging, FRAP or photoactive can be used to further study the mechanisms regulating the transport, and the effect of pathological stimulation on the transport. These results can help us understand whether MNTs play some role in the occurrence and development of some diseases.

In summary, there are still many more questions in the young field of MNTs, and better understanding of these questions may reveal new targets for treatment of diseases.

This work was supported by the National Basic Research Program of China (2013CB933701), the Projects of International Cooperation and Exchanges of the National Natural Science Foundation of China (30910103902), and the National Natural Science Foundation of China (81270159).

1 Rustom A, Saffrich R, Markovic I, et al. Nanotubular highways for intercellular organelle transport. Science, 2004, 303: 1007-1010

2 Watkins S C, Salter R D. Functional connectivity between immune cells mediated by tunneling nanotubules. Immunity, 2005, 23: 309-318

3 Koyanagi M, Brandes R P, Haendeler J, et al. Cell-to-cell connection of endothelial progenitor cells with cardiac myocytes by nanotubes: a novel mechanism for cell fate changes? Circ Res, 2005, 96: 1039-1041

4 Davis D M, Sowinski S. Membrane nanotubes: dynamic long- distance connections between animal cells. Nat Rev Mol Cell Biol, 2008, 9: 431-436

5 He K, Shi X, Zhang X, et al. Long-distance intercellular connectivity between cardiomyocytes and cardiofibroblasts mediated by membrane nanotubes. Cardiovasc Res, 2011, 92: 39-47

6 Chinnery H R, Pearlman E, Mcmenamin P G. Cutting edge: membrane nanotubes in vivo: a feature of MHC class II+ cells in the mouse cornea. J Immunol, 2008, 180: 5779-5783

7 Lou E, Fujisawa S, Barlas A, et al. Tunneling nanotubes: a new paradigm for studying intercellular communication and therapeutics in cancer. Commun Integr Biol, 2012, 5: 399-403

8 Kwok R. Cell biology: the new cell anatomy. Nature, 2011, 480: 26-28

9 Onfelt B, Nedvetzki S, Yanagi K, et al. Cutting edge: membrane nanotubes connect immune cells. J Immunol, 2004, 173: 1511-1513

10 Onfelt B, Nedvetzki S, Benninger R K, et al. Structurally distinct membrane nanotubes between human macrophages support long-distance vesicular traffic or surfing of bacteria. J Immunol, 2006, 177: 8476-8483

11 Comerci C J, Mace E M, Banerjee P P, et al. CD2 promotes human natural killer cell membrane nanotube formation. PLoS ONE, 2012, 7 : e47664

12 Wang X, Gerdes H H. Long-distance electrical coupling via tunneling nanotubes. Biochim Biophys Acta, 2012, 1818: 2082-2086

13 Chauveau A, Aucher A, Eissmann P, et al. Membrane nanotubes facilitate long-distance interactions between natural killer cells and target cells. Proc Natl Acad Sci USA, 2010, 107: 5545-5550

14 Wang X, Bukoreshtliev N V, Gerdes H H. Developing neurons form transient nanotubes facilitating electrical coupling and calcium signaling with distant astrocytes. PLoS ONE, 2012, 7: e47429

15 Wang X, Veruki M L, Bukoreshtliev N V, et al. Animal cells connected by nanotubes can be electrically coupled through interposed gap-junction channels. Proc Natl Acad Sci USA, 2010, 107: 17194-17199

16 Sowinski S, Jolly C, Berninghausen O, et al. Membrane nanotubes physically connect $\mathrm{T}$ cells over long distances presenting a novel route for HIV-1 transmission. Nat Cell Biol, 2008, 10: 211-219

17 Bukoreshtliev N V, Wang X, Hodneland E, et al. Selective block of tunneling nanotube (TNT) formation inhibits intercellular organelle transfer between PC12 cells. FEBS Lett, 2009, 583: 1481-1488

18 Luchetti F, Canonico B, Arcangeletti M, et al. Fas signalling promotes intercellular communication in T cells. PLoS ONE, 2012, 7: e35766

19 Seyed-Razavi Y, Hickey M J, Kuffova L, et al. Membrane nanotubes in myeloid cells in the adult mouse cornea represent a novel mode of immune cell interaction. Immunol Cell Biol, 2013, 91: 89-95

20 Wang Y, Cui J, Sun X, et al. Tunneling-nanotube development in astrocytes depends on p53 activation. Cell Death Differ, 2011, 18: $732-742$

21 Sun X, Wang Y, Zhang J, et al. Tunneling-nanotube direction determination in neurons and astrocytes. Cell Death Dis, 2012, 3: e438

22 Hase K, Kimura S, Takatsu H, et al. M-Sec promotes membrane nanotube formation by interacting with Ral and the exocyst complex. Nat Cell Biol, 2009, 11: 1427-1432

23 Vallabhaneni K, Haller H, Dumler I. Vascular smooth muscle cells initiate proliferation of mesenchymal stem cells by mitochondria transfer via tunneling nanotubes. Stem Cells Dev, 2012, 21: 3104-3113

24 Plotnikov E Y, Khryapenkova T G, Vasileva A K, et al. Cell-to-cell cross-talk between mesenchymal stem cells and cardiomyocytes in co-culture. J Cell Mol Med, 2008, 12: 1622-1631

25 He K, Luo W, Zhang Y, et al. Intercellular transportation of quantum dots mediated by membrane nanotubes. ACS Nano, 2010, 4: 3015-3022

26 Kuznetsov A V. Modeling bidirectional transport of quantum dot nanoparticles in membrane nanotubes. Math Biosci, 2011, 232: 101-109 
27 Domhan S, Ma L, Tai A, et al. Intercellular communication by exchange of cytoplasmic material via tunneling nano-tube like structures in primary human renal epithelial cells. PLoS ONE, 2011, 6: e21283

28 Ferrati S, Shamsudeen S, Summers H D, et al. Inter-endothelial transport of microvectors using cellular shuttles and tunneling nanotubes. Small, 2012, 8: 3151-3160

29 Plotnikov E Y, Khryapenkova T G, Galkina S I, et al. Cytoplasm and organelle transfer between mesenchymal multipotent stromal cells and renal tubular cells in co-culture. Exp Cell Res, 2010, 316: 2447-2455

30 Yasuda K, Park H C, Ratliff B, et al. Adriamycin nephropathy: a failure of endothelial progenitor cell-induced repair. Am J Pathol, 2010, 176: 1685-1695

31 Jolly C, Kashefi K, Hollinshead M, et al. HIV-1 cell to cell transfer across an Env-induced, actin-dependent synapse. J Exp Med, 2004, 199: 283-293

32 Chen P, Hubner W, Spinelli M A, et al. Predominant mode of human immunodeficiency virus transfer between $\mathrm{T}$ cells is mediated by sustained Env-dependent neutralization-resistant virological synapses. J Virol, 2007, 81: 12582-12595

33 Takahashi A, Kukita A, Li Y J, et al. Tunneling nanotube formation is essential for the regulation of osteoclastogenesis. J Cell Biochem, 2012, 114: 1238-1247

Open Access This article is distributed under the terms of the Creative Commons Attribution License which permits any use, distribution, and reproduction in any medium, provided the original author(s) and source are credited. 